\title{
Foreword: inverse problems in biology
}

\author{
Matti Lassas • Samuli Siltanen
}

Received: 6 November 2012 / Revised: 6 November 2012 / Published online: 5 June 2013

(C) Springer-Verlag Berlin Heidelberg 2013

Advances in the understanding of biology are often based on successful interpretation of indirect measurements. Those measurements may take the form of microscopic images, electrical neurophysiology data, X-ray microtomography reconstructions, observations of the end products of chemical reactions, or spatial distribution of species.

Sometimes the quantity of interest causes only a small and noise-sensitive effect to the measured data, which makes the interpretation task an ill-posed inverse problem. Generally, inverse problems are defined as the problem of recovering information from indirect, incomplete or noisy observations with a mathematical model. Both theoretical and computational inversion methods have developed tremendously in recent years. The purpose of the present Special Issue is to promote the application of the newest inversion methods to studies in mathematical biology, hopefully giving a boost to both fields.

The collection of articles in this special issue covers a wide spectrum of topics connecting the mathematics of biology and of inverse problems. Included are results related to population dynamics, therapeutic strategies for diseases, tumor growth, and analysis of models for heart and neural cables. Furthermore, there are studies of inverse problems appearing in the information recovery in molecular biology, protein polymerization, and chemical dynamics in neurons. Methodologically, the present articles combine several mathematical tools used in inverse problems, such as statistical methods, control theory, and parameter estimation, and apply them to both classical and novel models used in mathematical biology.

M. Lassas $(\bowtie) \cdot$ S. Siltanen

Department of Mathematics and Statistics, University of Helsinki, 00014 Helsinki, Finland e-mail: matti.lassas@helsinki.fi 